\title{
An Unexpected Cause of Diffuse Alveolar Hemorrhage in a Kidney Transplant Patient
}

\author{
Manuel Schlageter ${ }^{a}$ Kathleen D. Jahn ${ }^{b}$ Alexandar Tzankov ${ }^{a}$ Mark Wiese $^{c}$ \\ Lukas Bubendorfa $^{a}$ Michael Tamm ${ }^{b}$ Spasenija Savic ${ }^{a}$ \\ a Institute for Pathology, ${ }^{b}$ Clinic of Pneumology, and ${ }^{c}$ Clinic of Thoracic Surgery, University Hospital Basel, \\ Basel, Switzerland
}

\section{Established Facts}

- Angiosarcoma is a rare cause of diffuse alveolar hemorrhage and typically shows multiple nodules on chest CT scan.

\section{Novel Insights}

- Diffuse alveolar hemorrhage is rarely the presenting manifestation of metastasized angiosarcoma and can lack the typical CT findings of a metastatic malignant tumor.

\section{Key Words}

Diffuse alveolar hemorrhage · Angiosarcoma - Renal transplantation $\cdot$ Immunosuppression

\begin{abstract}
Diffuse alveolar hemorrhage (DAH) is a life-threatening condition requiring urgent treatment. There are many different treatment-relevant causes of $\mathrm{DAH}$, making the diagnostic approach to these patients complex and necessitating a multidisciplinary team. We report the case of a kidney transplant recipient in whom all diagnostic efforts did not reveal the cause of DAH, and only autopsy was able to establish an unexpected diagnosis.

(c) 2014 S. Karger AG, Basel
\end{abstract}

\section{Introduction}

Diffuse alveolar hemorrhage (DAH) is a life-threatening condition requiring urgent treatment. There are many different treatment-relevant causes of DAH which make correct diagnosis a complex matter, thus necessitating a multidisciplinary approach. Vasculitis syndromes are the most common causes of DAH, but nonimmunological etiologies do occur and must be considered in immunocompromised patients $[1,2]$. We report the case of a kidney transplant recipient where all the efforts at diagnosis did not reveal the cause of his DAH and the unexpected diagnosis was only established by autopsy.

\section{KARGER}

E-Mail karger@karger.com www.karger.com/res
(C) 2014 S. Karger AG, Basel

0025-7931/14/0876-0504\$39.50/0
Manuel Schlageter

Institute for Pathology, University Hospital Basel

Schönbeinstrasse 40

$\mathrm{CH}-4031$ Basel (Switzerland)

E-Mail Manuel.schlageter@usb.ch 


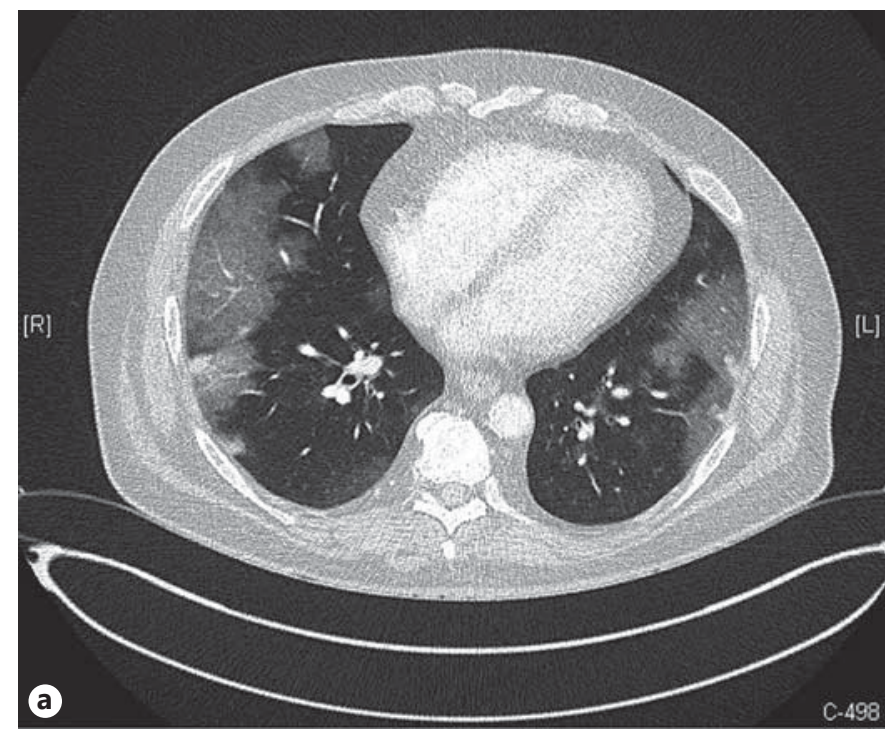

Fig. 1. a Chest CT scan with bilateral ground-glass opacities in the lung periphery and subpleural consolidations, well-compatible with DAH. b Histological slide of the wedge resection with DAH showing intra-alveolar accumulation of blood. HE $\times 100$. Upon histological examination, the subpleural consolidations represented areas of subacute DAH with proliferation of intra-alveolar fibromyxoid tissue (image not shown). c Histological slide of the lung examined at autopsy with pulmonary metastasis of the angiosarcoma with nodular proliferation of irregular, interanastomosing vascular spaces. Adjacent lung with DAH. $\mathrm{HE} \times 200$.

\section{Case Report}

A 63-year-old male patient was admitted to hospital due to acute hemoptysis with severe dyspnea. He had been well until then. He had undergone kidney transplantation 2 years earlier for chronic renal failure due to diabetic and vascular nephropathy. Immunosuppression followed, in the form of tacrolimus and mycophenolate mofetil administration. There had not been any postoperative complications.

The chest CT scan upon admission revealed areas of bilateral ground-glass opacities, compatible with DAH (fig. 1a). Blood tests showed elevated C-reactive protein $(81 \mathrm{mg} / \mathrm{l}$, reference range $<10$ $\mathrm{mg} / \mathrm{l})$ with only a slight leukocytosis $\left(10.3 \times 10^{9} / \mathrm{l}\right.$, reference range $3.50-10.00 \times 10^{9} / 1$ ) and a drop in hemoglobin level (from 163 to $133 \mathrm{~g} / \mathrm{l}$, reference range $140-180 \mathrm{~g} / \mathrm{l}$ ) compared to his previous measurement 4 months earlier. Platelet count, coagulation studies and tests of the complement system were normal. ANA, ANCA
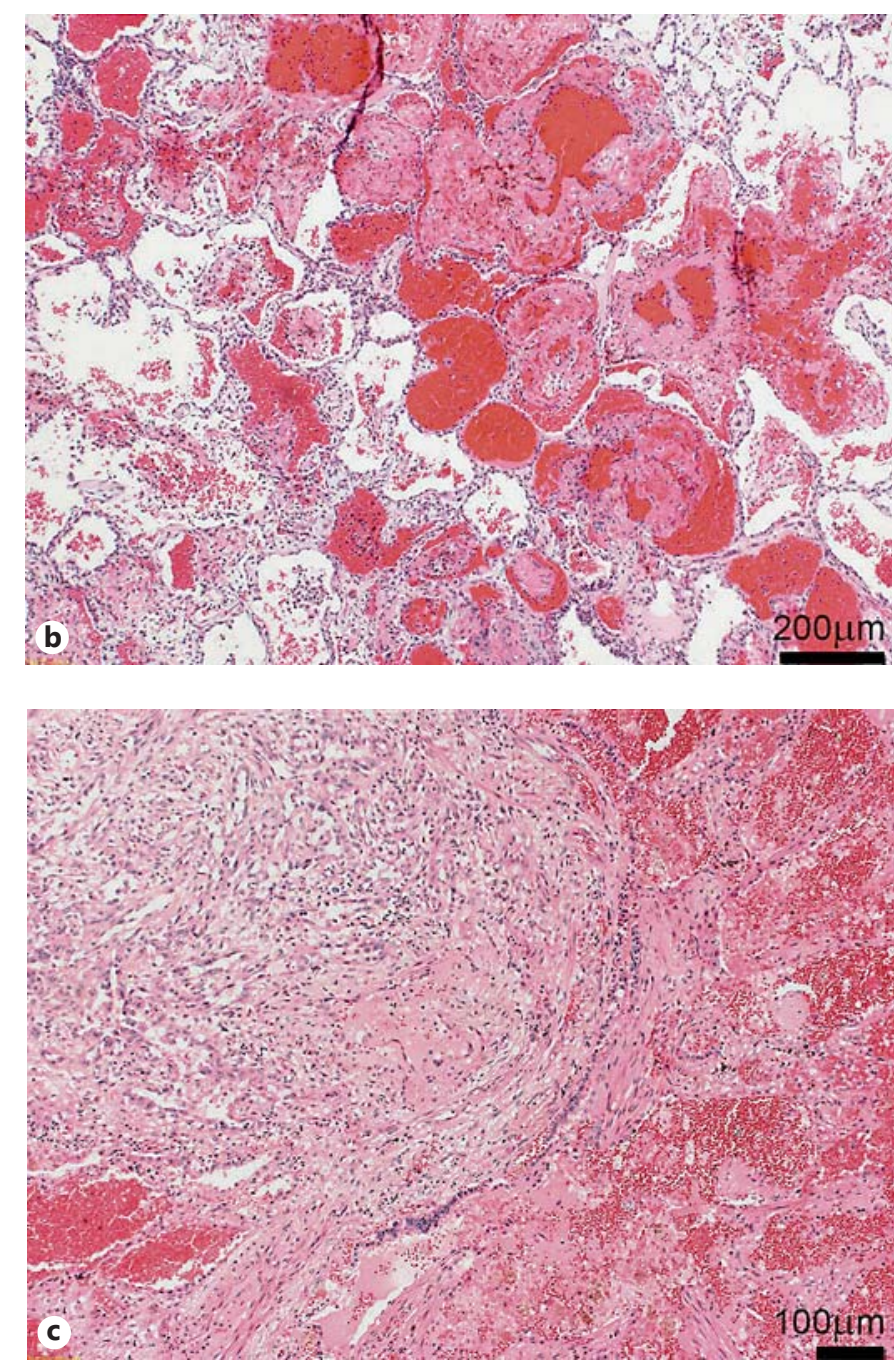

and anti-GBM were within the normal range. The renal parameters and urine tests were inconspicuous.

Bronchoscopy with bronchoalveolar lavage fluid was performed and showed the classic features of a diffuse hemorrhage. Special stains, cultures and immunofluorescence of the bloody bronchoalveolar lavage fluid were negative for bacteria, mycobacteria, fungi, Pneumocystis jirovecii, Legionella pneumophila, cytomegalovirus and other viruses. A multiplex PCR for the detection of different viruses and bacteria (Respifinder ${ }^{\circledR}$, PathoFinder B.V., Maastricht, The Netherlands) was also negative. Microbiological culture of the bronchoalveolar lavage fluid detected only sporadic colonies of Aspergillus fumigatus and the blood cultures were negative. Nevertheless, an infectious cause of DAH was deemed to be the most likely, due to the immunosuppression, and an empiric broad-spectrum antibiotic and antifungal therapy was administered. Immunosuppression was reduced by stopping mycophenolate mofetil and adding corticosteroids. When the serum Aspergil- 
lus antigen turned out to be negative, antifungal treatment was stopped.

As the hemoptysis did not improve with treatment, a videoassisted thoracoscopic wedge resection of the lung was performed. Histological examination showed acute and subacute DAH with no signs of infection, neutrophilic capillaritis, vasculitis or malignancy (fig. 1b). PCR for mycobacteria, panfungal PCR and microbiological cultures of the lung tissue were all negative.

After eliminating infection as a possible cause, an immunologically mediated or drug-induced DAH was considered to be the most likely and treatment with high-dose prednisone was initiated. The long-term immunosuppression regimen was changed to prednisone and cyclophosphamide. Tacrolimus was discontinued. The hemoptysis and the general condition of the patient improved dramatically and he was discharged from hospital.

One month later, he developed hypercalcemia and had to be rehospitalized. The hemoptysis had not stopped, but was only occasional and mild. The patient reported a swelling of his left forearm, where he had had an arteriovenous fistula (AVF) for the past 6 years. Radiologically, the left ulna and radius were fractured and showed severe osteopenia, compatible with Sudeck's osteodystrophy. The fracture was stabilized with a cast. One week later, the AVF was surgically removed because of thrombosis and a bone biopsy of the fractured radius was taken, which showed only the fracture site without signs of malignancy. The removed AVF was not submitted for histological examination.

The patient's general condition deteriorated rapidly and blood tests showed elevated inflammatory parameters. A chest CT scan revealed a right-sided pleural effusion with new bilateral, dense infiltrates and ground-glass opacities. Video-assisted thoracoscopic surgery was performed on suspicion of an empyema and a lung biopsy was taken. Histology showed an invasive pulmonary aspergillosis with acute and subacute DAH in the background. Antifungal treatment was immediately started, but the patient died only 3 days after surgery.

Upon autopsy, the lung was rusty brown with areas of fresh hemorrhage and without focal pulmonary lesions. Histological examination confirmed bilateral angioinvasive aspergillosis of the lung. However, unexpectedly, pulmonary infiltrations of an angiosarcoma (AS) measuring up to $2 \mathrm{~mm}$ were also found (fig. 1c). The tumor was negative for human herpes virus 8 . The left, previously AVF-bearing forearm revealed the primary site of the AS with diffuse infiltration of the radius and ulna. Further metastases, measuring up to only $3 \mathrm{~mm}$, were present in the spleen and the pelvic bone marrow.

\section{Discussion}

$\mathrm{DAH}$ is a medical emergency requiring urgent treatment, often before an underlying cause can be established. Differential diagnosis depends on the immune status of the patient. In immunocompetent patients, the etiology of DAH is most commonly immune-mediated as in ANCA-associated vasculitis, Goodpasture's syndrome and connective tissue disease, and requires treatment with high-dose corticosteroids $[1,2]$. In immuno- compromised patients, DAH is far more commonly caused by infections, like invasive aspergillosis or cytomegalovirus pneumonitis [3]. Drugs and localized causes of pulmonary hemorrhage like bronchiectasis and malignancies, which can mimic DAH, must also be included in differential diagnoses. All of these were considered in the diagnostic work-up of our patient, but even invasive surgical lung biopsy was not able to reveal the underlying cause.

After an extensive search for bacterial, fungal and viral causative agents and an unsuccessful empiric antibiotic and antifungal treatment, an infectious cause could be excluded and an immunologically mediated DAH was postulated. Indeed, on high-dose corticosteroids, the patient's general condition improved and the hemoptysis decreased in amount and frequency, although it did not stop completely. Unfortunately, 8 weeks after the administration of high-dose corticosteroid treatment, the patient died of invasive pulmonary aspergillosis, a complication of the immunosuppression. Autopsy finally revealed the etiology of the DAH by showing metastasized AS with minute pulmonary metastases.

Pulmonary metastasized AS is a rare cause of DAH typically showing multiple nodules on chest CT [4]. DAH is rarely the presenting manifestation of metastasized AS and can lack the typical CT findings of a metastatic malignant tumor, as was the case in this patient [5]. In this setting, open lung biopsy usually establishes the diagnosis. Both wedge resections of the patient were completely embedded and were histologically analyzed. The metastases were not present due to sampling error, as they were nonpalpable and widely dispersed. Intriguingly, the patient's hemoptysis improved under high-dose corticosteroid treatment, a finding that has been reported once before [6].

Rarely, with less than 20 cases having been reported so far, AS develops within or adjacent to an AVF after renal transplantation and it can also occur in immunocompetent patients [7-9]. AVF-related AS can occur 2-25 years after renal transplantation and it has a poor prognosis. Regular examination of AVFs should be performed, with any new symptoms or clinical findings prompting imaging studies, and histological examination should also be considered to allow for an early diagnosis. In our case, only a radial bone biopsy of the fracture site was sent for histological examination, and again, sampling error precluded a diagnosis. Histological examination of the surgically removed shunt would have led to the correct diagnosis, but, unfortunately, this was not submitted for further analysis. 
In conclusion, although every diagnostic effort was undertaken to find the cause of this patient's DAH, the pulmonary metastasized AS was eventually only detected by autopsy. This case demonstrates the importance of a correct diagnosis in order to administer the appropriate treatment in immunosuppressed patients with DAH and the value of autopsy to confirm and complete a clinical diagnosis. Even if the typical findings on CT are lacking, metastatic AS must be considered in a patient with a DAH and an AVF.

\section{Acknowledgements}

We thank Prof. M. Dickenmann, Clinic of Nephrology, University Hospital Basel, for reviewing the manuscript.

\section{References}

1 Krause ML, Cartin-Ceba R, Specks U, Peikert $\mathrm{T}$ : Update on diffuse alveolar hemorrhage and pulmonary vasculitis. Immunol Allergy Clin North Am 2012;32:587-600.

2 Collard HR, Schwarz MI: Diffuse alveolar hemorrhage. Clin Chest Med 2004;25:583592.

3 von Ranke FM, Zanetti G, Hochhegger B, Marchiori E: Infectious diseases causing diffuse alveolar hemorrhage in immunocompetent patients: a state-of-the-art review. Lung 2012;191:9-18.
4 Tateishi U, Hasegawa T, Kusumoto M, Yamazaki N, Iinuma G, Muramatsu Y, Moriyama N: Metastatic angiosarcoma of the lung: spectrum of CT findings. AJR Am J Roentgenol 2003;180:1671-1674.

5 Adem C, Aubry MC, Tazelaar HD, Myers JL: Metastatic angiosarcoma masquerading as diffuse pulmonary hemorrhage: clinicopathologic analysis of 7 new patients. Arch Pathol Lab Med 2001;125:1562-1565.

$\checkmark 6$ Rajdev N, Green R, Crosby WH: Angiosarcoma with pulmonary siderosis and persistent reticulocytosis. Steroid responsiveness suggests an immune basis. Arch Intern Med 1978;138:1549-1551.
Webster P, Wujanto L, Fisher C, Walker M, Ramakrishnan R, Naresh K, Thomas JM, Papalois V, Crane J, Taube D, Duncan N: Malignancies confined to disused arteriovenous fistulae in renal transplant patients: an important differential diagnosis. Am J Nephrol 2011;34:42-48.

-8 Murata S, Kaneko S, Kusatake K, Furumura M, Sakieda K, Harada Y, Maruyama R, Morita $\mathrm{E}$ : Angiosarcoma of the forearm arising in an arteriovenous fistula in a renal transplant recipient. Eur J Dermatol 2011;21:792-793.

9 Chanyaputhipong J, Hock DL, Sebastian MG: Disseminated angiosarcoma of the dialysis fistula in 2 patients without kidney transplants. Am J Kidney Dis 2011;57:917-920. 